\title{
Characterizing the Micromechanical Properties of Myeloblasts from Cancer Patients with Optical Tweezers
}

\author{
Youhua Tan, Student Member, IEEE, Anskar Y.H. Leung, Kaiqun Wang, Tsz-Kan Fung, and Dong Sun, \\ Senior Member, IEEE
}

\begin{abstract}
Cell mechanics, in particular mechanical properties, has been suggested as a new biomarker indicative of cell state and phenotype. Acute myeloid leukemia (AML) is characterized by the abnormal increase of myeloblasts in blood and bone marrow. While AML has been extensively studied from the perspectives of biochemical and genetic aspects, little is known about its cellular biophysical properties. In this study, optical tweezer technology was used to examine the micromechanical properties of myeloblasts from bone marrow of AML patients at single cell level. The myeloblasts were separately analyzed according to their expression of $\mathrm{CD}^{+} 4^{+}$, a marker of primitive hematopoietic cells. To extract the intrinsic properties from the relationship between the stretching force and the induced deformation, a theoretical approach was developed to model the mechanical responses of cells and further characterize their mechanical properties. The preliminary results show that the area compressibility modulus of $\mathrm{CD}^{+}{ }^{+}$myeloblasts was significantly less than that of $\mathrm{CD34}^{-}$cells, which indicate that micromechanical properties are unique features of myeloblasts and provide us with an insight into the cell mechanics of primitive AML cells.
\end{abstract}

\section{INTRODUCTION}

Cell mechanics determines how a cell senses and responds to external or internal mechanical stimuli and plays an important role in regulating cellular functions, such as cell growth, migration, differentiation, division, and apoptosis [1]. Cell mechanics, in particular mechanical properties, is mainly determined by cellular cytoskeleton network. Any changes in cytoskeleton may lead to changes in cell mechanics and further result in development of numerous diseases and abnormal mechanotransduction [2]. Accumulating evidence has suggested that cell mechanical properties may be associated with the onset and progression with some diseases [3], [4]. For example, red blood cells (RBC) obtained from patients with sickle cell disease or infected by plasmodium falciparum displayed lower deformability and sticky behaviors [5], [6],

This work was supported by a grant from Research Grants Council of the Hong Kong Special Administrative Region, China [Reference No. CityU 120310], UGC Special Equipment Grant [SEG_CityU 01], and a grant from City University of Hong Kong [Reference No. 9360131].

Y. Tan, K. Wang, and D. Sun are with the Department of Manufacturing Engineering and Engineering Management, City University of Hong Kong, Hong Kong, China (e-mail: youhuatan2@cityu.edu.hk, kaiwang6@student.cityu.edu.hk, medsun@cityu.edu.hk).

A. Y. H. Leung and K. Fung are with the Department of Medicine, the University of Hong Kong, Hong Kong, China (e-mail: ayhleung@hkucc.hku.hk, fungkan@hkucc.hku.hk). which led to abnormal microcirculation. Metastatic cancer cells were demonstrated to be more than $70 \%$ softer than their benign counterparts [7]. Prostate cancer cells with different metastatic potentials exhibited distinct Young's moduli [8]. Moreover, micromechanical properties may be indication of cell phenotype. For instance, cardiac cells exhibited significantly higher viscoelastic modulus than skeletal muscle cells [9], [10].

Acute myeloid leukemia (AML) is characterized by the abnormal increase of myeloblasts in blood and bone marrow [11]. Induction chemotherapy is the mainstay of treatment. However, mortality of AML patients is high and few can be cured and survive in the long term [12]. AML is considered to be initiated by unchecked growth of immature cells as a result of accumulated mutations. Recent studies suggest that leukemic cells are heterogenous and their biological functions are diverse among the population [13]-[16]. For example, AML $\mathrm{CD}_{4} 4^{+}$cells exhibited more resistance to apoptosis than $\mathrm{CD} 34^{-}$ fractions [13]. $\mathrm{CD} 34^{+} \mathrm{CD} 38^{-}$leukemia progenitors had increased chemotherapy resistance, decreased immunogenicity and dendritic cell transformation capacities compared to $\mathrm{CD} 34^{+} \mathrm{CD} 38^{+}$cells [15]. The primitive $\mathrm{CD} 34^{+}$cells displayed higher sensitivity to the cholesterol synthesis inhibitor lovastatin than CD34 cells [16]. However, the underlying mechanisms are still not fully understood. Currently, study on diseases mainly focuses on the molecular, microbiological, immunological and pathological aspects. The connection between cell mechanics and diseases, such as AML, remains elusive. While AML has been characterized by morphologic, karyotypic and more recently genetic features [17], [18], information pertaining to its cellular biophysical properties is completely lacking.

Optical tweezers provide the ability to apply force and deformation on a micro-scaled object on the order of picoNewton $\left(p N, 10^{-12} \mathrm{~N}\right)$ and nanometer $\left(\mathrm{nm}, 10^{-9} \mathrm{~m}\right)$, respectively. This technology is utilized in this study to manipulate primitive myeloblasts from AML patients for biomechanical properties characterization. Cells are stretched by progressively increasing the distance between the beads. Through force calibration and image processing, the relationship between the stretching force and the induced cell deformation can be established, from which the biomechanical properties of CD34 cells are characterized based on our cell 
mechanical model [19], [20]. The findings in this study will provide insights into the cell mechanics of primitive AML hematopoietic cells.

\section{Cell MechanicAl Model}

In cell stretching, micro-beads were attached to cell surfaces serving as handles. A cell was stretched by progressively increasing the distance between the beads. We assumed that the shape of the suspended cell before deformation was spherical. Because of the symmetry of both cell shape and imposed force, the shape of the cell after optical stretching was rotationally symmetric. The problem of mechanical modeling was then simplified to be two-dimensional, as shown in Fig. 1.

Membrane theory has been used to describe the deformation behaviors during cell manipulation, such as optical stretching and cell injection [19]-[22]. A biological cell, especially a suspended cell, was usually simplified to be a pressurized liquid drop enveloped by a spherical biomembrane [19]-[23], which was represented by a thin layer of membrane. Cellular resistance to external forces could be described by membrane theory. The equilibrium equations were used to represent the force balance of the cell membrane, expressed as follows

$$
\begin{aligned}
& \frac{\partial T_{m}}{\partial \lambda_{m}} \lambda_{m}{ }^{\prime}+\frac{\partial T_{m}}{\partial \lambda_{c}} \lambda_{c}^{\prime}=\frac{r^{\prime}}{r}\left(T_{c}-T_{m}\right) \\
& K_{m} T_{m}+K_{c} T_{c}=\sigma_{n}
\end{aligned}
$$

where $\lambda_{m}$ and $\lambda_{c}, T_{m}$ and $T_{c}$, and $K_{m}$ and $K_{c}$ are the principal stretch ratios, tensions and curvatures, respectively. $\sigma_{n}$ was the pressure normally acting on the membrane. The definitions of all the quantities can be found in [19]-[22].

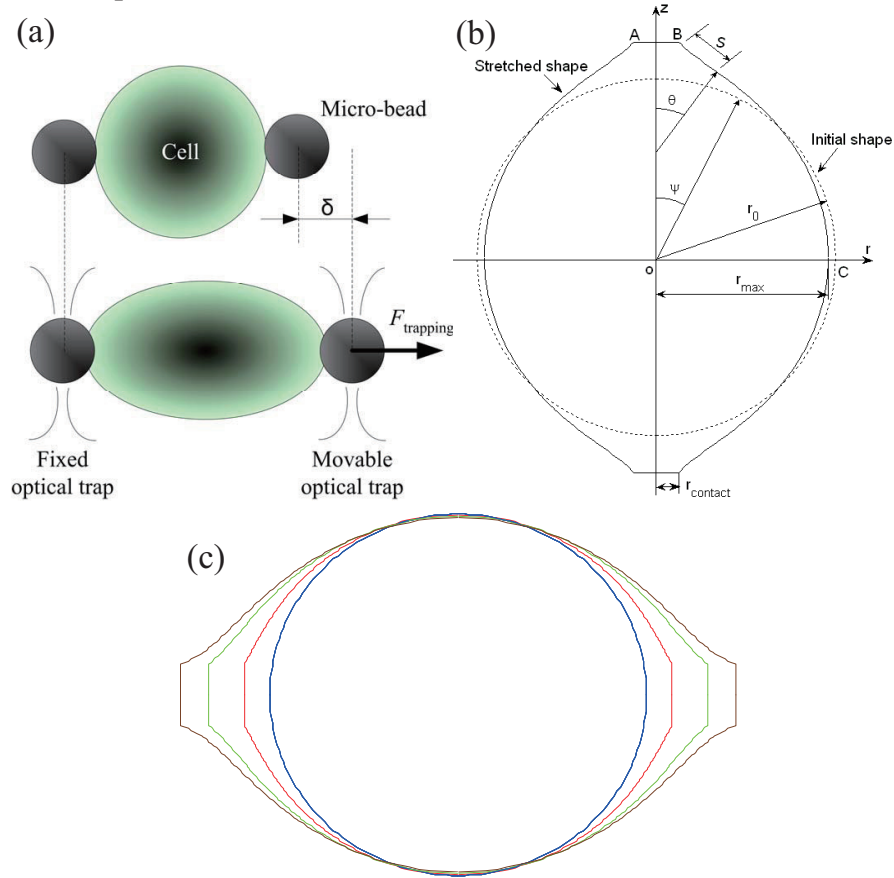

Fig. 1. (a) Schematic of cell stretching, (b) Coordinate definition for biological cells before and after optical stretching, and (c) calculated deformed cell shapes, where the blue line denotes the initial spherical cell shape.

The constitutive material proposed by Evans and Skalak was used to represent the deformation characteristics of cell membranes [24]. We assumed that the membrane tensions were uniform and isotropic, the membrane tensions $T_{m}$ and $T_{c}$ could be derived as follows [24]

$$
T_{m}=T_{c}=k_{\alpha} \alpha
$$

where $k_{\alpha}$ is the area compressibility modulus. $\alpha$ is the area dilation ratio defined by $\alpha=\left(A-A_{0}\right) / A_{0}$, where $A_{0}$ and $A$ are the areas of the cell surface before and after stretching.

Given the biomechanical property $k_{\alpha}$, the equilibrium equations (1) and (2) can be solved numerically [19], [20]. The stretching force and the cell deformation can be then calculated as well as the deformed cell shapes (Fig. 1c). Thereafter, the deformation-force relationship is established.

\section{Cell Stretching EXPERIMENTS}

\section{A. Experimental System}

Fig. 2 shows the optical tweezer system (BioRyx 200, Arryx), which mainly includes a multiple optical trap system, an inverted optical microscope (Nikon TE2000, Japan), an $X-Y-Z$ motorized stage (ProScan, Prior Scientific), and a CCD system. A continuous wave laser beam (YLM-3-1064-LP-AX1, IPG Photonics) with wavelength of $1064 \mathrm{~nm}$ is transmitted via the optical fiber and sculpted by the holographic optical tweezer (HOT) device. Multiple optical traps are then created on the observation plane. Positions of optical traps can be controlled individually in three dimensions by the HOT device through modulating the phase distribution of a spatial light modulator. These well-controlled traps serve as micro end-effectors to manipulate multiple micro/nano-scaled objects with size ranged from $100 \mathrm{~nm}$ to $100 \mu \mathrm{m}$. The sample is contained in a home-built chamber, which is placed on the motorized stage with a positioning accuracy of $40 \mathrm{~nm}$. The manipulation process is monitored by the CCD detection system and positions of the targets are obtained through image processing.

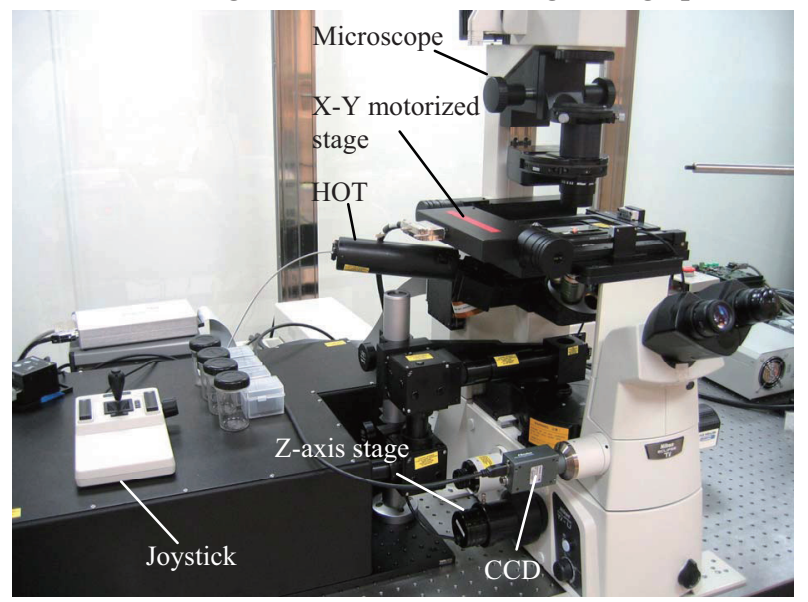

Fig. 2. Robotic manipulation system with optical tweezers (HOT: holographic optical tweezer).

\section{B. Experimental Preparation}

With informed consent, myeloblasts were isolated and purified from the bone marrow of patients with AML. 
According to the standard procedures [25], these cells were fractionated in terms of $\mathrm{CD}_{3} 4^{+}$expression, a marker of primitive hematopoietic cells.

Before experiments, micro-beads were attached to the cell surface. The detailed procedure was given elsewhere [20]. In brief, streptavidin-coated polystyrene beads (Bangs Lab., Fishers, IN) were incubated with biotinylated concanavalin A (ConA, Sigma) at $4^{\circ} \mathrm{C}$. The treated beads were then mixed with cell suspension to create cell-bead attachment.

The viscous-drag-force calibration method was adopted here to evaluate the optical forces. When a bead was driven in a flow by an optical trap, the escaping force was equal to the viscous force calculated according to Stokes' law [20], [26]. The relationship between laser power and optical force was established through calibration.

\section{Cell Stretching}
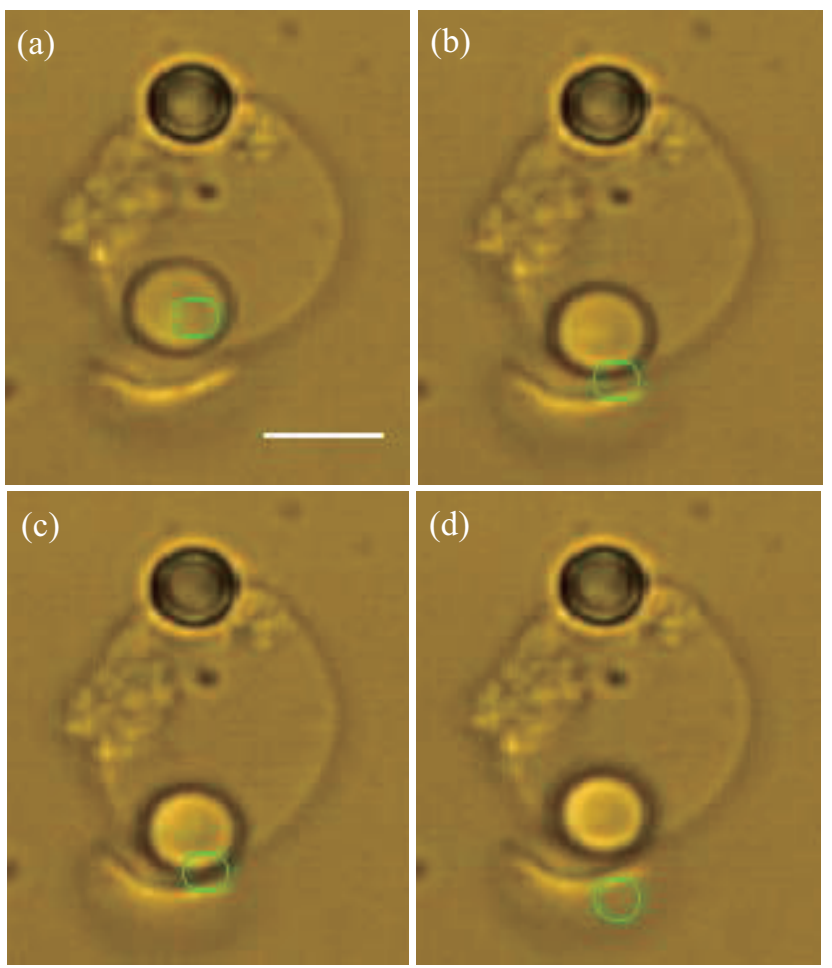

Fig. 3. Process of cell stretching. (a) Before stretching, (b) during stretching, (c) stretching to the maximum deformation, and (d) the bead escapes the trap. The scale bar is $5 \mu \mathrm{m}$.

The diluted cell-bead mixture was injected into the chamber for stretching experiments. Fig. 3 illustrates the process of cell stretching. The cell with two beads bound to the opposite sides in diameter or with one side attached to the chamber and the other side bound to a bead was chosen for test. With one bead fixed by an optical trap, the other bead was grasped and moved by another trap. The moving direction was determined along the joint line passing the centroids of the two beads. The cell was stretched by progressively increasing the distance between the beads. When the deviation between cell and bead exceeded a critical value, the trapping force decreased dramatically. Then the bead escaped the trap, as shown in Fig. 3 (d). By adjusting laser power, various optical forces could be obtained from the calibrated force-power relationship. Note that cell deformation was measured from the image where the cell was stretched to the maximum deformation and further stretch would lead to bead escape from the trap, as shown in Fig. 3 (c). Therefore, the relationship between the stretching force and the cell deformation was then established. We performed several times of cell stretching tests on each cell. It was found in experiments that some cells exhibited stiffening characteristics after repetitive stretching (more than five times). To exclude this influence, the experimental data in the first three times tests were utilized in this paper.
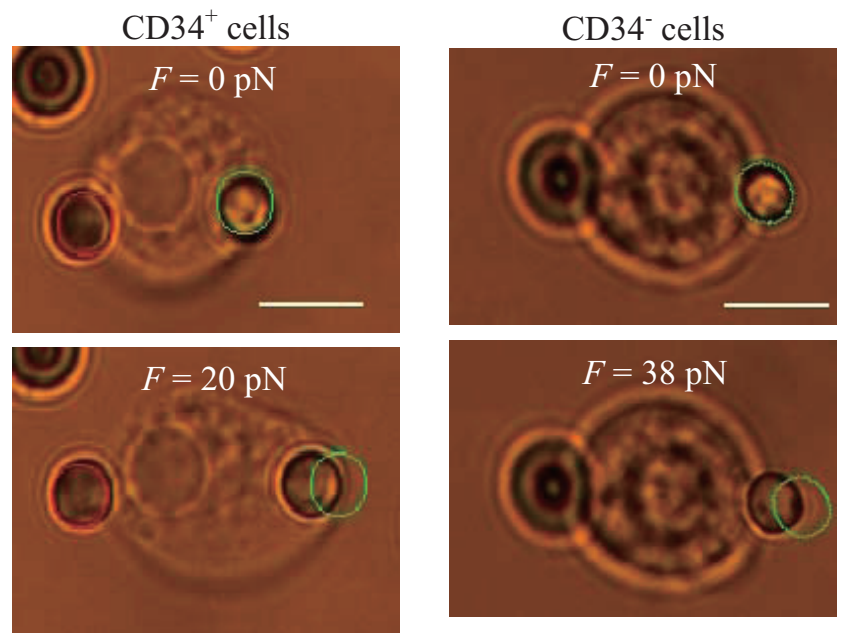

Fig. 4. Different deformation behaviors of $\mathrm{CD} 34^{+}$and $\mathrm{CD} 34^{-}$cells from patients with AML. The scale bar is $5 \mu m$.

\section{RESUltS AND Discussions}

We measured the mechanical stiffness of myeloblasts from AML patients separately in terms of $\mathrm{CD} 34^{+}$expression. The experiments were repeated three times, where 62 71 CD34 ${ }^{+}$ cells and 57 63 CD34 cells were tested in total during the stretching experiments and the results were averaged.

In experiments, no significant difference was found in the cell size of $\mathrm{CD} 34^{+/-}$population, i.e., $10.1 \pm 1.0 \mu \mathrm{m}$ and $10.6 \pm 1.4$ $\mu m$ for the diameter of $\mathrm{CD} 34^{+}$and $\mathrm{CD} 34^{-}$cells, respectively. Typical deformation responses of $\mathrm{CD} 34^{+/-}$cells under different stretching forces are shown in Fig. 4. Increasing membrane stress was exerted on the cell surface as the cell was stretched. The deformation of AML CD34 ${ }^{+}$cells is much larger than that of CD34- cells, which suggests that there exists significant difference in the deformability of AML CD $34^{+}$and CD $34^{-}$cells. The difference in deformability indicates that AML cells with different phenotypes exhibit distinct biomechanical properties.

Fig. 5 shows the quantitative analysis of the deformation behaviors of AML CD34 $4^{+/}$cells. It can be seen that the deformation of $\mathrm{CD} 34^{+}$cells varies over a relatively wide range compared to that of $\mathrm{CD}^{-} 4^{-}$cells. This finding may suggest that AML CD $34^{+}$fraction may contain multiple subpopulations, which are heterogeneous from the perspective of cell mechanics, in particular, cell deformability. Nevertheless, all the measurement results show that the induced deformation of CD $34^{+}$cells is greater than $\mathrm{CD}^{-}$cells under the same stretching force, indicating that the primitive $\mathrm{CD}_{3} 4^{+}$cells are 
significantly softer than $\mathrm{CD} 34^{-}$cells, i.e., $\mathrm{CD} 34^{+}$cells exhibit much higher deformability. Additionally, both $\mathrm{CD} 34^{+}$and CD34 cells displayed two-phase deformation behavior. In the first phase, AML CD $34^{+/-}$cells responded quasi-linearly to the small optical force. When the force increased to some extent, AML myeloblasts exhibited 'saturated' mechanical characteristics, i.e., the cell deformation entered into a plateau and the growth of the deformation lowered down. To evaluate the biomechanical properties of AML myeloblasts, the cell mechanical model described above was applied. The modeling deformation behaviors in cell stretching are also shown in Fig. 5. Based on this model, the area compressibility modulus $k_{\alpha}$, was characterized as $0.25 \pm 0.15 \mathrm{~N} / \mathrm{m}$ and $1.40 \pm 0.71 \mathrm{~N} / \mathrm{m}$ for $\mathrm{CD}_{4} 4^{+}$and $\mathrm{CD} 34^{-}$myeloblasts, respectively. This finding indicates that AML CD34 cells are significantly stiffer than $\mathrm{CD}_{3} 4^{+}$cells, which may be related to their unique functions of these two AML cell populations. Recent reports have confirmed the presence of leukemic stem cells (LSC) in the population of $\mathrm{CD} 34^{+}$cells [14]. The significance of this finding in relation to leukemogenesis particularly at the LSC level would have to be further examined.

\begin{tabular}{|r|l|l|l|l|l|l|}
\hline $\begin{array}{r}\text { Force } \\
(\mathrm{pN})\end{array}$ & 0 & 14.2 & 21.0 & 27.8 & 34.7 & 41.5 \\
\hline $\begin{array}{c}\text { AML CD34 } \\
\text { cells }\end{array}$ & 0 & 1.83 & 2.71 & 3.53 & 3.85 & 4.36 \\
\hline $\begin{array}{c}\text { SD }\left(\mathrm{CD} 34^{+}\right. \\
\text {cells })\end{array}$ & 0 & 0.65 & 1.03 & 1.34 & 1.62 & 1.86 \\
\hline $\begin{array}{c}\text { AML CD34- } \\
\text { cells }\end{array}$ & 0 & 0.32 & 0.51 & 0.86 & 1.10 & 1.33 \\
\hline $\begin{array}{c}\text { SD }\left(\mathrm{CD} 34^{-}\right. \\
\text {cells })\end{array}$ & 0 & 0.23 & 0.32 & 0.51 & 0.52 & 0.53 \\
\hline
\end{tabular}

SD: standard deviation. The values in the table exclusive of the first row denote the cell deformations under various stretching forces and the unit is $\mu \mathrm{m}$.

\section{CONCLUSIONS}

In summary, this paper presented our latest results of mechanical characterization of myeloblasts from patients with AML. Optical tweezers were used to examine the mechanical properties of AML cells in terms of CD34 $4^{+}$expression. A cell mechanical model was developed to interpret the deformation behaviors of cells, from which the area compressibility modulus of cells was characterized. The results showed that CD $34^{+}$cells exhibited higher deformability than CD34 cells. The connection between this finding and leukemogenesis needs to be further investigated in future.

\section{REFERENCES}

[1] G. Bao and S. Suresh, "Cell and molecular mechanics of biological materials," Nat. Mater., vol. 2, pp. 715-725, 2003.

[2] D. E. Ingber, "Mechanobiology and diseases of mechanotransduction," Ann. Med., vol. 35, pp. 564-577, 2003.

[3] S. Suresh, J. Spatz, J. P. Mills, A. Micoulet, M. Dao, C. T. Lim, M. Beil, and T. Seufferlein, "Connections between single-cell biomechanics and human disease states: gastrointestinal cancer and malaria," Acta Biomater., vol. 1, pp. 15-30, 2005.

[4] G. Y. H. Lee and C. T. Lim, "Biomechanics approaches to studying human diseases," Trends Biotechnol., vol. 25, pp. 111-118, 2007.
[5] G. B. Nash, C. S. Johnson, and H. J. Meiselman, "Mechanical properties of oxygenated red blood cells in sickle cell (HbSS) disease," Blood, vol. 63, pp. 73-82, 1984 ..

[6] F. K. Glenister, R. L. Coppel, A. F. Cowman, N. Mohandas, and B. M. Cooke, "Contribution of parasite proteins to altered mechanical properties of malaria-infected red blood cells," Blood, vol. 99, pp. 1060-1063, 2002.

[7] S. E. Cross, Y. S. Jin, J. Y. Rao, and J. K. Gimzewski, "Nanomechanical analysis of cells from cancer patients," Nat. Nanotechnol., vol. 2, pp. 780-783, 2007.

[8] E. C. Faria, N. Ma, E. Gazi, P. Gardner, M. Brown, N. W. Clarke, and R. D. Snook, "Measurement of elastic properties of prostate cancer cells using AFM," Analyst, vol. 133, pp. 1498-1500, 2008.

[9] A. B. Mathur, A. M. Collinsworth, W. M. Reichert, W. E. Kraus, and G. A. Truskey, "Endothelial, cardiac muscle and skeletal muscle exhibit different viscous and elastic properties as determined by atomic force microscopy," J. Biomech., vol.34, pp. 1545-1553, 2001.

[10] D. Fish, J. Orenstein, and S. Bloom, "Passive stiffness of isolated cardiac and skeletal myocytes in the hamster," Circ. Res., vol. 54, pp. 267-276, 1984.

[11] B. Lowenberg, J. R. Downing, and A. Burnett, "Acute myeloid leukaemia,” N. Engl. J. Med., vol. 341, pp. 1051-1062, 1999.

[12] F. Ravandi, A. K. Burnett, E. D. Agura, and H. M. Kantarjian, "Progress in the treatment of acute myeloid leukemia," Cancer, vol. 110, pp. 1900-1910, 2007.

[13] A. van Stijn, M. A. van der Pol, A. Kok, P. M. Montje, G. M. J. M. Roemen, R. H. J. Beelen, G. J. Ossenkoppele, and G. J. Schuurhuis, "Differences between the CD34+ and CD34- blast compartments in apoptosis resistance in acute myeloid leukemia," Haemotologica, vol. 88, pp. 497-508, 2003.

[14] D. Bonnet, "Normal and leukaemic stem cells," Br. J. Haematol., vol. 130, pp. 469-479, 2005.

[15] R. T. Costello, F. Mallet, B. Gaugler, D. Sainty, C. Arnoulet, J-A. Gastaut, and D. Olive, "Human acute myeloid leukemia CD34+/38- progenitor cells have decreased sensitivity to chemotherapy and fas-induced apoptosis, reduced immunogenicity, and impaired dendritic cell transformation capacities," Cancer Res., vol. 60, pp. 4403-4411, 2000.

[16] S. D. P. W. M. de Jonge-Peeters, K. van der Weide, F. Kuipers, W. J. Sluiter, E. G. E. de Vries, and E. Vellenga, "Variability in responsiveness to lovastatin of the primitive CD34+ AML subfraction compared to normal CD34+ cells," Ann. Hematol., vol. 88, pp. 573-580, 2009.

[17] D. H. Ryan, "Phenotypic heterogeneity in acute leukemia," Clin. Chim. Acta, vol. 206, pp. 9-23, 1992.

[18] M. A. Bitter, M. M. Le Beau, J. D. Rowley, R. A. Larson, H. M. Golomb, and J. W. Vardiman, "Associations between morphology, karyotype, and clinical features in myeloid leukemia," Hum. Pathol., vol. 18, pp. 211-225, 1987.

[19] Y. Tan, D. Sun, W. Huang, and S. H. Cheng, "Mechanical modeling of biological cells in microinjection," IEEE Trans. NanoBioSci., vol. 7, pp. 257-266, 2008.

[20] Y. Tan, D. Sun, J. Wang, and W. Huang, "Mechanical characterization of human red blood cells under different osmotic conditions by robotic manipulation with optical tweezers," IEEE Trans. Biomed. Eng., vol. 57, pp. 1816-1825, 2010.

[21] Y. Tan, D. Sun, and W. Huang, "Mechanical modeling of red blood cells during optical stretching," J. Biomech. Eng.-Trans. ASME, vol. 132, pp. 044504, 2010.

[22] Y. Tan, D. Sun, W. Huang, and S. H. Cheng, "Characterizing Mechanical Properties of Biological Cells by Microinjection," IEEE Trans. NanoBioSci., vol. 9, pp. 171-180, 2010.

[23] Z. Zhang, M. A. Ferenczi, and C. R. Thomas, "A micromanipulation technique with a theoretical cell model for determining mechanical properties of single mammalian cells," Chem. Eng. Sci., vol. 47, pp. 1347-1354, 1992.

[24] E. A. Evans, and R. Skalak, Mechanics and thermodynamics of biomembranes, CRC Press, 1980.

[25] A. Cheung, H. Chow, R. Liang, and A. Leung, "A comparative study of bone marrow and peripheral blood CD34+ myeloblasts in acute myeloid leukaemia," Br. J. Haematol., vol. 144, pp. 484-491, 2008. 